TRANSACTIONS OF THE

AMERICAN MATHEMATICAL SOCIETY

Volume 173, November 1972

\title{
ON THE GENUS OF A GROUP
}

\author{
BY \\ ARTHUR T. WHITE $\left({ }^{1}\right)$
}

\begin{abstract}
The genus of a group is defined to be the minimum genus for any Cayley color graph of the group. All finite planar groups have been determined, but little is known about the genus of finite nonplanar groups. In this paper two families of toroidal groups are presented; the genus is calculated for certain abelian groups; and upper bounds are given for the genera of the symmetric and alternating groups and for some hamiltonian groups.
\end{abstract}

Introduction. Corresponding to a presentation $P$, in terms of generators and relations, for a group $G$ is the Cayley color graph $D_{P}(G)$, defined as follows: The vertices of $D_{P}(G)$ are the elements of $G$; with the generators are associated distinct colors, and there is a directed edge in $D_{P}(G)$ from $g_{1}$ to $g_{2}$ and colored with the color of generator $h$, if and only if $g_{1} b=g_{2}$ in $G$. For each generator of order two, we adopt the standard convention of replacing each pair of directed edges $\left(g_{1}, g_{2}\right)$ and $\left(g_{2}, g_{1}\right)$ by the single undirected edge $\left[g_{1}, g_{2}\right]$. The genus, $\gamma$, of $a$ graph is the minimum genus among the genera of all closed orientable 2-manifolds in which the graph can be imbedded. The genus of a Cayley color graph, $\gamma\left(D_{P}(G)\right)$, is the genus of the graph that results when all arrows and colors are omitted from $D_{P}(G)$. We define the genus of a group $G$ to be the minimum genus for any Cayley color graph of $G$; i.e.

$$
\gamma(G)=\min _{\text {over } P} \gamma\left(D_{P}(G)\right)
$$

If $G$ has no finite genus, we write $\gamma(G)=\infty$. Levinson [10] has shown that an infinite group $G$ has either $\gamma(G)=0$ or $\gamma(G)=\infty$. Here we consider only finite groups. A minimal presentation has no redundant generators. If $\gamma(G)=\gamma\left(D_{P}(G)\right)$, we call $P$ a genus presentation for $G$.

Dyck [7] (see also Burnside [4, Chapters 18 and 19]) considered maps on closed orientable 2-manifolds that are transformed into themselves in accordance with a fixed group $G$, acting transitively on the regions of the map. Any such map gives an upper bound for $\gamma(G)$, as a "dual" formed in terms of Burnside's white regions gives a Cayley color graph for $G$. Brahana [3] studied groups represented by regular maps on closed orientable 2-manifolds; these maps correspond to presentations on two generators, one of which is of order two. In this context the

Presented to the Society, January 18, 1972; received by the editors September 13, 1971. $20 \mathrm{~F} 10,55 \mathrm{~A} 15$.

Key words and phrases. Graph, group, generators and relations, Cayley color graph of a group, imbedding, genus of a graph, genus of a group.

(1) Research supported in part by Western Michigan University (Faculty Research Fellowship). 
group acts transitively on the edges of the map, and again an upper bound for $\gamma(G)$ is obtained. In this paper we regard $G$ as acting transitively on the vertices of the map induced by imbedding $D_{P}(G)$ in a closed orientable 2-manifold; indeed, it is well-known that the automorphism group of $D_{P}(G)$ is isomorphic to $G$, independent of the presentation $P$. Our point of view may give a closed orientable 2-manifold of lower genus for a given group; for example the direct product $Z_{2} \times Z_{4}$ (where $Z_{n}$ denotes the cyclic group of order $n$ ) is toroidal for Dyck (or Burnside) and for Brahana, yet $\gamma\left(Z_{2} \times Z_{4}\right)=0$; see Figure 1. Also, our definition has the following intuitive appeal: a Cayley color graph gives a "picture" of its group, from which many group properties (such as commutivity, normality of certain subgroups, the multiplication table) can be discerned; perhaps then it is natural to seek the most efficient surface upon which to "draw" this picture.

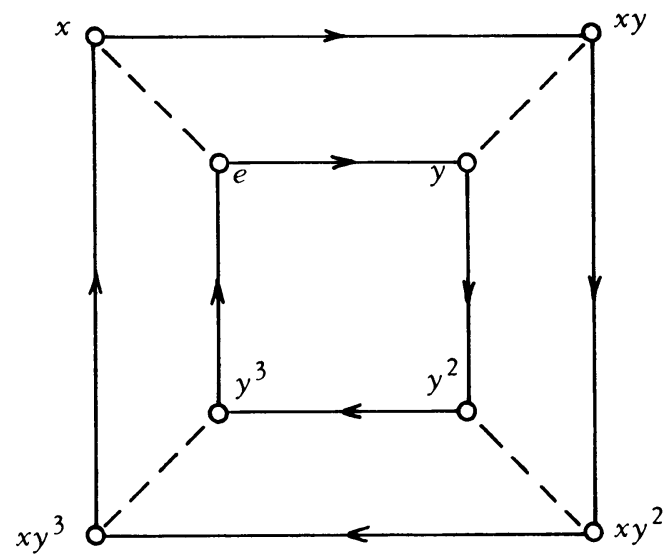

Figure 1. $Z_{2} \times Z_{4}=\left\langle x, y \mid x^{2}=y^{4}=x y x y^{-1}=e\right\rangle$

Planar groups. Maschke [11] determined all finite planar Cayley color graphs, and thus all finite groups of genus zero. We summarize his findings (see [5, p. 37] to identify Maschke's extended octahedral and extended icosahedral groups as $Z_{2} \times S_{4}$ and $Z_{2} \times S_{5}$, respectively):

Theorem 1. The finite group $G$ is planar if and only if $G=G_{1} \times G_{2}$, where $G_{1}=Z_{1}$ or $Z_{2}$ and $G_{2}=Z_{n}, D_{n}, A_{4}, S_{4}$ or $A_{5}$.

In this paper we present two infinite families of toroidal groups, determine the genus for certain abelian groups, and derive upper bounds for the genera of several other classes of groups. We will find particularly useful an algebraic description of graphical imbeddings developed by Edmonds [8] (see also Youngs [15]).

Edmonds' permutation technique. Let a connected graph $H$ have vertex set $V(H)=\left\{v_{1}, v_{2}, \cdots, v_{n}\right\}$. Let $V(i)=\left\{v_{j} \mid v_{i} v_{j} \in E(H)\right\}$, where $E(H)$ denotes the edge set of $H$. Let $p_{i}: V(i) \rightarrow V(i)$ be a cyclic permutation, of length $|V(i)|$; then 
$\left(p_{1}, p_{2}, \cdots, p_{n}\right)$ uniquely determines a 2 -cell imbedding of $H$ in a closed orientable 2-manifold such that the adjacencies at $v_{i}$ are given (in the order imposed by the fixed orientation) by $p_{i}$. Conversely, corresponding to a 2-cell imbedding of a connected graph $H$, the $p_{i}$ are uniquely determined. Furthermore, let $D=\left\{\left(v_{i}, v_{j}\right)\right\}$ $\left.v_{i} v_{j} \in E(H)\right\}$, and define $F: D \rightarrow D$ by $F\left(v_{i}, v_{j}\right)=\left(v_{j}, p_{v_{j}}\left(v_{i}\right)\right)$; then the orbits under the permutation $F$ correspond to the region boundaries of the imbedding. We will specify each such orbit by listing the constituent vertices in sequence. In the present context, of course, the vertices are group elements.

Two classes of toroidal groups. We are now prepared to compute the genus for two infinite families of groups.

Theorem 2. The group $Z_{m} \times Z_{n}$ is toroidal if $(m, n) \geq 3$ and planar otberwise.

Proof. If $(m, n)=1, Z_{m} \times Z_{n}=Z_{m n}$. If $(m, n)=2, Z_{m} \times Z_{n}=Z_{2} \times Z_{m n / 2}$. For $(m, n) \geq 3, \gamma\left(Z_{m} \times Z_{n}\right) \geq 1$, since $Z_{m} \times Z_{n}$ is not one of the groups of Theorem 1. Now consider the following presentation $P$ :

$$
Z_{m} \times Z_{n}=\left\langle r, s \mid r^{m}=s^{n}=r s r^{-1} s^{-1}=e\right\rangle ;
$$

then $\gamma\left(Z_{m} \times Z_{n}\right) \leq \gamma\left(D_{P}\left(Z_{m} \times Z_{n}\right)\right)=1$ (see Figure 2 for the case $m=n=3$; the generalization to arbitrary $m, n \geq 3$ is entirely obvious).

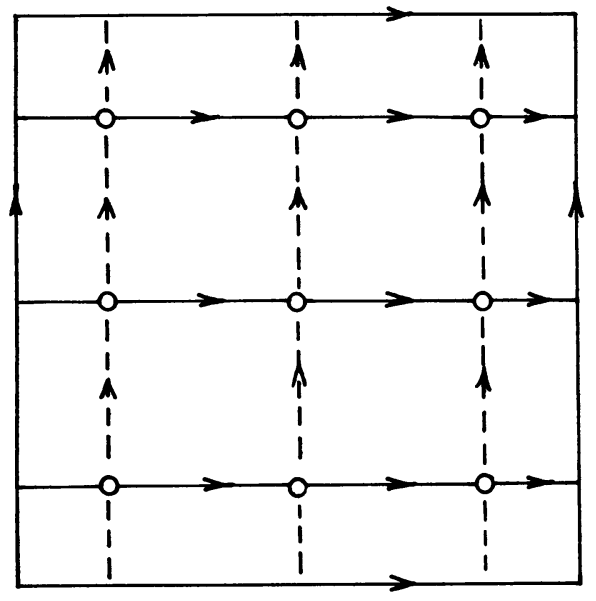

Figure 2. $Z_{3} \times Z_{3}$ on the torus

The next class of groups includes the quaternions, the smallest order nonplanar group.

Theorem 3. Let $G_{n}$ be the dicyclic group:

$$
G_{n}=\left\langle x, y \mid x^{2 n}=x^{n} y^{-2}=y^{-1} x y x=e\right\rangle ;
$$

then $\gamma\left(G_{n}\right)=1$, for $n>1$. 
Proof. We note that $\gamma\left(G_{n}\right) \geq 1$, since $G_{n}$ is not planar for $n>1\left(G_{1}=Z_{4}\right)$. We show that $\gamma\left(D_{P}\left(G_{n}\right)\right) \leq 1$ for $P$ as in the statement of the theorem. Let $V_{1}=$ $\left\{x^{i} \mid 1 \leq i \leq 2 n\right\}$ and $V_{2}=\left\{x^{i} y \mid 1 \leq i \leq 2 n\right\}$; then $G_{n}=V_{1} \cup V_{2}$, a disjoint union. Now in $D_{P}\left(G_{n}\right)$ every edge colored $x$ joins two vertices in $V_{1}$ or two vertices in $V_{2}$, since $\left(x^{i}\right) x \in V_{1}$ and $\left(x^{j} y\right) x \in V_{2}$, for otherwise $\left(x^{j} y\right) x=x^{k}$, for some $k$, and $y$ would be redundant. Also, every edge colored $y$ joins a vertex in $V_{1}$ to a vertex in $V_{2}$ (or vice-versa), since $\left(x^{i}\right) y \in V_{2}$ and $\left(x^{j} y\right) y=x^{j}\left(y^{2}\right)=x^{j} x^{n}=x^{j+n} \in V_{1}$. We select the permutations $p_{g}$ of Edmonds' algorithm as follows:

$$
p_{g}= \begin{cases}\left(g x^{-1}, g y^{-1}, g x, g y\right) & \text { if } g \in V_{1}, \\ \left(g y, g x, g y^{-1}, g x^{-1}\right) & \text { if } g \in V_{2} .\end{cases}
$$

Figure 3 depicts the situation at $g \in V_{2}$.

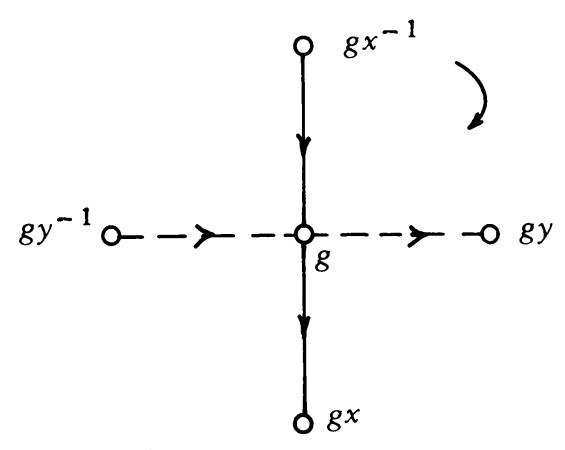

Figure 3. The situation at $g \in V_{2}$

We now compute orbits under $F$. Consider the directed edge $\left(a, a y^{-1}\right)$, where $a \in V_{1}$. Then $a y^{-1} \in V_{2}$, and we compute the next vertex in this orbit to be $p_{a y-1}(a)=a y^{-1} x \in V_{2}$. Continuing in this fashion, we obtain the orbit: $a-a y^{-1}$ $a y^{-1} x-a y^{-1} x y-a y^{-1} x y x-a y^{-1} x y x y^{-1} \ldots$. But $a y^{-1} x y x=a$ and $a y^{-1} x y x y^{-1}=a y^{-1}$, so this orbit has length four. We obtain $2 n$ distinct orbits of this form, as $a$ ranges over $V_{1}$. Similarly, we obtain $2 n$ additional distinct orbits, all of length four, containing directed edges of the form $\left(a, a y^{-1}\right)$, where $a \in V_{2}$. This exhausts all the directed edges of $D_{P}\left(G_{n}\right)$, so that this 2-cell imbedding has $p=4 n, q=8 n$, and $r=4 n$ (where $p, q$, and $r$ denote the number of vertices, edges, and regions respectively). The euler formula $p-q+r=2-2 \gamma$ now gives $\gamma=1$.

Abelian groups. The following theorem is a standard result in elementary group theory; see for example [2, p. 348].

Theorem 4. Let $G$ be an abelian group of order $n$; then $G$ can be expressed (uniquely) as a direct product:

$$
G=Z_{m_{1}} \times Z_{m_{2}} \times \cdots \times Z_{m_{r}}
$$


where $n=\Pi_{i=1}^{r} m_{i}, m_{i}$ is a multiple of $m_{i+1}$ for $1 \leq i \leq r-1$, and $r$ is the rank of $G$.

There is a graphical product that corresponds in a natural manner to the direct product for groups. Let $H_{1}$ and $H_{2}$ be two graphs, with vertex sets $V\left(H_{i}\right)$ and edge sets $E\left(H_{i}\right), i=1,2$ respectively. The cartesian product $H_{1} \times H_{2}$ has:

(i) $V\left(H_{1} \times H_{2}\right)=V\left(H_{1}\right) \times V\left(H_{2}\right)$,

(ii) $E\left(H_{1} \times H_{2}\right)=\left\{\left[\left(u_{1}, u_{2}\right),\left(v_{1}, v_{2}\right)\right] \mid u_{1}=v_{1}\right.$ and $\left[u_{2}, v_{2}\right] \in E\left(H_{2}\right)$, or $u_{2}=v_{2}$ and $\left.\left[u_{1}, v_{1}\right] \in E\left(H_{1}\right)\right\}$.

Similarly, the cartesian product $D_{P_{1}}\left(G_{1}\right) \times D_{P_{2}}\left(G_{2}\right)$ of two Cayley color graphs has vertex set $V\left(D_{P_{1}}\left(G_{1}\right)\right) \times V\left(D_{P_{2}}\left(G_{2}\right)\right)$, and $\left(g_{1}, g_{2}\right)$ is joined to $\left(g_{1}^{\prime}, g_{2}^{\prime}\right)$ by an edge colored $b$ if and only if either:

(i) $g_{1}=g_{1}^{\prime}$ and $g_{2} b=g_{2}^{\prime}$, for $b$ a generator in $P_{2}$, or

(ii) $g_{2}=g_{2}^{\prime}$ and $g_{1} b=g_{1}^{\prime}$, for $b$ a generator in $P_{1}$.

Now let the presentations $P_{i}$ for $G_{i}, i=1,2$, be given by:

$$
\begin{aligned}
& G_{1}=\left\langle k_{1}, \cdots, k_{m} \mid w_{1}=\cdots=w_{r}=e\right\rangle, \\
& G_{2}=\left\langle k_{m+1}, \cdots, k_{n} \mid w_{r+1}=\cdots=w_{r+s}=e\right\rangle ;
\end{aligned}
$$

then the (external) direct product $G_{1} \times G_{2}$ has standard presentation $P$, given by:

$$
G_{1} \times G_{2}=\left\langle k_{1}, \cdots, k_{n} \mid w_{1}=\cdots=w_{r+s}=k_{i} k_{j} k_{i}^{-1} k_{j}^{-1}=e, 1 \leq i \leq m<j \leq n\right\rangle,
$$

where we represent $g_{1} \in G_{1}$ as $\left(g_{1}, e_{G_{2}}\right)$ and $g_{2} \in G_{2}$ as $\left(e_{G_{1}}, g_{2}\right)$, in the product $G_{1} \times G_{2}$.

The next theorem follows directly from these definitions. (Figure 1 illustrates the theorem, for $G_{1}=Z_{2}, G_{2}=Z_{4}$.)

Theorem 5. The cartesian product of the Cayley color graphs for two groups is a Cayley color graph for the direct product of the two groups; i.e.

$$
D_{P}\left(G_{1} \times G_{2}\right)=D_{P_{1}}\left(G_{1}\right) \times D_{P_{2}}\left(G_{2}\right) .
$$

Let $C_{m_{i}}$ denote a directed cycle with all edges colored with color $i$. Then if $Z_{m_{i}}=\left\langle x_{i} \mid x_{i}^{m_{i}}=e\right\rangle$ is given by presentation $P_{i}, D_{P_{i}}\left(Z_{m_{i}}\right)=C_{m_{i}}$, and Theorem 4, together with repeated applications of Theorem 5 , gives: then

Corollary 5a. If $G$ is an abelian group of rank $r, G=Z_{m_{1}} \times Z_{m_{2}} \times \cdots \times Z_{m_{r}}$,

$$
D_{P}(G)=C_{m_{1}} \times C_{m_{2}} \times \cdots \times C_{m_{r}} .
$$

Thus the genus of an abelian group is no greater than the genus of an appropriate repeated cartesian product of cycles. In [14], the author has investigated the genus of repeated cartesian products of cycles. Here we consider first the case where $m_{i}=2,1 \leq i \leq r ; C_{2}$ denotes the graph having two vertices and one edge, corresponding to the group $Z_{2}$. The following lemma is useful. 
Lemma 1. Suppose that 3 does not divide the order of the group $G$, and let $P$ be a minimal presentation for $G$. Then $D_{P}(G)$ contains no triangles.

Proof. Suppose $D_{P}(G)$ contains a triangle; then we find a closed walk $b_{1}^{a_{1}} b_{2}^{a_{2}} b_{3}^{a_{3}}=e$ in $D_{P}(G)$, where $b_{i}$ is a generator in $P$, and $a_{i}= \pm 1$. If any two of the $b_{i}$ are distinct, then one of the se two is redundant, contradicting the minimality of $P$. If, on the other hand, $b_{1}=b_{2}=b_{3}$, then the $a_{i}$ all have the same sign; but then $b_{1}^{3}=e$, so that 3 divides the order of $G$.

Theorem 6. Let $G_{r}$ be the group of order $2^{r}$ baving every nonidentity element of order 2. Then

$$
\gamma\left(G_{r}\right)=1+2^{r-3}(r-4), \quad r \geq 2 .
$$

Proof. $G_{r}$ is necessarily abelian, of rank $r$. By Lemma 1 , an imbedding of a Cayley color graph for $G_{r}$ giving the genus will have no triangular regions. Hence by a well-known consequence of the euler formula (see, for example $[9$, p. 118]),

$$
\gamma\left(G_{r}\right) \geq 1-p / 2+q / 4
$$

where $p=2^{r}$ and $2 q \geq r 2^{r}$; thus

$$
\gamma\left(G_{r}\right) \geq 1-2^{r-1}+r 2^{r-3}=1+2^{r-3}(r-4) .
$$

But $G_{r}$ is the group of Corollary 5a, with all $m_{i}=2$, so that

$$
\gamma\left(G_{r}\right) \leq \gamma\left(D_{P}\left(G_{r}\right)\right)=\gamma\left(Q_{r}\right)=1+2^{r-3}(r-4) ;
$$

where the genus of the $r$-cube $Q$, has been computed ([1] and [12]).

We next generalize this result, in accordance with [14], where the following theorem is established:

Theorem 7. Let the graph $H_{n}$ be defined by: $H_{1}=C_{2 m_{1}} ; H_{n}=H_{n-1} \times C_{2 m_{n}}$, $n \geq 2$. If $m_{i} \geq 2,1 \leq i \leq n$, then

$$
\gamma\left(H_{n}\right)=1+2^{n-2}(n-2) \prod_{i=1}^{n} m_{i}
$$

The constructive method employed in the proof of Theorem 7 is easily modified to allow any or all of the $m_{i}$ to be 1 ; suppose $m_{i} \geqq 2$ for $1 \leqq i \leqq k$, but $m_{i}=1$ for $k+1 \leq i \leq n$. Then we obtain:

Theorem 7a. $\gamma\left(H_{n}\right)=1+2^{n-3}(n+k-4) \Pi_{i=1}^{n} m_{i}$.

This leads directly to:

Theorem 8. Let $G$ be an abelian group of rank $r$, written as

$$
G=Z_{2 m_{1}} \times Z_{2 m_{2}} \times \cdots \times Z_{2 m_{r}},
$$

where $m_{i}$ is a multiple of $m_{i+1}$ for $1 \leq i \leq r-1$. 
Suppose that $m_{k} \neq 1$, but $m_{k+1}=1,0 \leq k \leq r$. Then

$$
\gamma(G)=1+2^{r-3}(r+k-4) \prod_{i=1}^{r} m_{i} .
$$

Proof. By Corollary $5 \mathrm{a}$ and Theorem $7 \mathrm{a}$,

$$
\begin{aligned}
\gamma(G) & \leq \gamma\left(D_{P}(G)\right)=\gamma\left(C_{2 m_{1}} \times C_{2 m_{2}} \times \cdots \times C_{2 m_{r}}\right) \\
& =1+2^{r-3}(r+k-4) \prod_{i=1}^{r} m_{i} .
\end{aligned}
$$

The imbedding constructed for Theorem $7 \mathrm{a}$ has every region bounded by exactly four sides, and is minimal for $G$ (since $G$ has rank $r$ ), unless another imbedding can be found involving triangular regions. But any such imbedding must be of a Cayley color graph for $G$ having larger degree than that corresponding to presentation $P$, and routine manipulations of the euler formula establish that the genus is then no longer minimal. Hence

$$
\gamma(G) \geq 1+2^{r-3}(r+k-4) \prod_{i=1}^{r} m_{i}
$$

Hamiltonian groups. A nonabelian group $G$ is said to be bamiltonian if every subgroup of $G$ is normal in $G$. For example, the quaternions $Q$ are hamiltonian. In fact, hamiltonian groups have been characterized [6]:

Theorem 9. A group $G$ is bamiltonian if and only if $G=Q \times A_{1} \times A_{2}$, where $A_{1}$ is abelian of odd order and $A_{2}$ bas every nonidentity element of order 2 .

Suppose that $A_{1}=Z_{m_{1}} \times Z_{m_{2}} \times \cdots \times Z_{m_{r}}$, with $r$ the rank of $A_{1}$, and that $A_{2}$ has order $2^{n}$, with $r \leq n$. Then we have:

Theorem 10. The bamiltonian group $G$ bas genus bounded by:

$$
\gamma(G) \leq 1+2^{n} \prod_{i=1}^{n} m_{i}(n+r) .
$$

Proof. Since $K_{4,4}$ (with edges colored and directed appropriately) is a Cayley color graph of minimum genus for $Q$, we apply Theorem 5 to see that $K_{4,4} \times$ $C_{2 m_{1}} \times \cdots \times C_{2 m_{r}} \times Q_{n-r}$ is a Cayley color graph for $G$. The constructive techniques of [14] can be further extended to achieve a quadrilateral imbedding of this graph, giving the upper bound.

Formulas for four classes of presentations. In this section we develop genus formulas for four classes of presentations; these formulas collectively describe minimal imbeddings for all planar groups, as well as upper bounds for the genera of the groups $S_{n}$ and $A_{n}$. 
Theorem 11. If $G$ is minimally generated by $\left\{g_{1}, \cdots, g_{n}\right\}$ and satisfies at least the relations $g^{m_{i}}=e=\left(\Pi_{j=1}^{n} g_{j}\right)^{k}, \quad 1 \leq i \leq n$, then

$$
\gamma(G) \leq 1+\frac{|G|}{2}\left(n-1-\frac{1}{k}-\sum_{j=1}^{n} \frac{1}{m_{j}}\right) .
$$

Proof. Select $p_{g}=\left(g g_{1}, g g_{1}^{-1}, g g_{2}, g g_{2}^{-1}, \cdots, g g_{n}, g g_{n}^{-1}\right)$, for all $g \in G$. Then, using Edmonds' algorithm, we compute orbits as follows:

(i) An orbit containing the directed edge $\left(a, a g_{i}^{-1}\right)$ continues with $p_{a g}{ }_{i}^{-1}(a)=$ $a g{ }_{i}^{-2}$; hence this orbit corresponds to the relation $g_{i}^{m_{i}}=e$ and has length $m_{i}$. (If $m_{i}=2$, we draw edges for both $g g_{i}=g^{\prime}$ and $g^{\prime} g_{i}=g$, obtaining $|G| / 2$ 2-sided regions; for each such region, the two sides may be identified and the arrows removed, so that the region is destroyed but the genus is unaffected.)

(ii) An orbit containing the directed edge $\left(a, a g_{i}\right)$ continues with $p_{a g_{i}}(a)=$ $a g_{i} g_{i+1}$; hence this orbit corresponds to the relation $\left(\Pi_{i=1}^{n} g_{i}\right)^{k}=e$ and has length $n k$. As there are no other orbits, we find:

$$
r=\sum_{i=1}^{n} r_{m_{i}}+r_{n k}=\sum_{i=1}^{n} \frac{|G|}{m_{i}}+\frac{|G|}{k},
$$

where $r_{m_{i}}$ denotes the number of $m_{i}$-sided regions in the imbedding corresponding to the relation $g_{i}^{m_{i}}=e$, and $r_{n k}$ denotes the number of $n k$-sided regions corresponding to the relation $\left(\Pi_{j=1}^{n} g_{j}\right)^{k}=e$. The euler formula now gives the genus.

We note that an equivalent formula was obtained by Burnside [4, p. 398] in a different context. Theorem 11 gives $\gamma(G)$ exactly, for $G=Z_{m}, A_{4}, S_{4}, A_{5}$, or $Z_{3} \times Z_{3}$. We also obtain the following two corollaries:

Corollary 11a.

$$
\gamma\left(S_{n}\right) \leq 1+\frac{(n-2) !}{4}\left(n^{2}-5 n+2\right), \quad n \geq 2 .
$$

Proof. Take $S_{n}=\langle s, t\rangle$, where $s=\left(\begin{array}{llll}1 & 2 & 3 & \cdots\end{array}\right), t=\left(\begin{array}{ll}1 & 2\end{array}\right)$; then $s^{n}=t^{2}=$ $(s t)^{n-1}=e$.

Corollary $11 b$.

$$
\gamma\left(A_{n}\right) \leq\left\{\begin{array}{l}
1+\frac{(n-1)(n-3) !}{8}\left(n^{2}-6 n+4\right), \quad n \text { odd }, \\
1+\frac{n(n-2) !(n-5)}{8}, \quad n \text { even. }
\end{array}\right.
$$

Proof. Take $A_{n}=\langle s, r\rangle$, where $s=(12 \cdots n-2), r=(1 n-1)(2 n)$ if $n$ is odd; then $s^{n-2}=r^{2}=(s r)^{n}=e$. If $n$ is even, let $A_{n}=\langle s, r\rangle$, where $s=(12 \cdots n-1)$ and $r=\left(\begin{array}{ll}1 & 2\end{array}\right)(3 n)$, so that $s^{n-1}=r^{2}=(s r)^{n-1}=e$ (see [3]).

The two formulas given above for $S_{n}$ and $A_{n}$ respectively were also found by Brahana [3], using a different method and in a slightly different context. 
Theorem 12. If $G$ is minimally generated by $\left\{g_{1}, \cdots, g_{n}\right\}$ and satisfies at least the relations $g_{i}^{2}=e=\left(g_{k} g_{k+1}\right)^{w_{k}}, 1 \leq i \leq n, 1 \leq k \leq n$; and if $D_{P}(G)$ is bipartite for this presentation $P$, then

$$
\gamma(G) \leq 1+\frac{|G|}{4}\left(n-2-\sum_{k=1}^{n} \frac{1}{w}_{k}\right) .
$$

Proof. Select

$$
p_{g}= \begin{cases}\left(g g_{1}, g g_{2}, \cdots, g g_{n}\right), & g \in V_{1}, \\ \left(g g_{n}, \cdots, g g_{2}, g g_{1}\right), & g \in V_{2},\end{cases}
$$

where $V_{1} \cup V_{2}$ is the partition of the vertex set of the bipartite graph $D_{P}(G)$. Then, computing orbits, we find:

$$
r=\sum_{k=1}^{n} r_{2 w_{k}}=\sum_{k=1}^{n} \frac{|G|}{2 w_{k}} ;
$$

the genus now follows from the euler formula.

In applying Theorem 12 , it is naturally desirable to order the $g_{i}$ so that $\sum_{k=1}^{n} 1 / w_{k}$ is a maximum. Theorem 12 gives $\gamma(G)$ exactly for $G=D_{m}, S_{4}, Z_{2} \times$ $D_{m}, Z_{2} \times S_{4}, Z_{2} \times A_{5}$, and the group $(3,3,3 ; 2)$ of Coxeter (see [5, p. 134]).

The corollary below gives a bound for $\gamma\left(S_{n}\right)$ that coincides with a result of Jacques; see [13].

Corollary 12a。 $\gamma\left(S_{n}\right) \leq 1+(n ! / 8)(n-5), \quad n \geq 6$.

Proof. $S_{n}=\left\langle\left(\begin{array}{ll}1 & 2\end{array}\right),\left(\begin{array}{l}2 \\ 2\end{array}\right),\left(\begin{array}{ll}3 & 4\end{array}\right), \cdots,(n-1 n)\right\rangle$; now take $V_{1}=A_{n}$.

Note that this bound is not as sharp as that of Corollary 11 a. 3, then

Theorem 13. If $G=\left\langle g_{1}, g_{2} \mid g_{1}^{m_{1}}=g_{2}^{m_{2}}=e=\left(g_{1} g_{2} g_{1}^{-1} g_{2}^{-1}\right)^{k}\right\rangle$, where $m_{1} \geq m_{2} \geq$

$$
\gamma(G) \leq 1+\frac{|G|}{2}\left(1-\frac{1}{k}\right)
$$

Proof. We use $p_{g}=\left(g g_{1}, g g_{2}, g g_{1}^{-1}, g g_{2}^{-1}\right)$ for all $g \in G$. Then every orbit has length $4 k$, so that $r=|G| / k, p=|G|, q=2|G|$, and the euler formula shows that

$$
\gamma(G) \leq \gamma\left(D_{P}(G)\right) \leq 1+\frac{|G|}{2}\left(1-\frac{1}{k}\right)
$$

where $P$ is the presentation given above for $G$.

We note that the group $G$ of Theorem 13 is abelian if and only if $k=1$, and that in this case we have $\gamma\left(Z_{m_{1}} \times Z_{m_{2}}\right) \leq 1$, in agreement with Theorem 2 .

We now vary the presentation of Theorem 13 by setting $m_{2}=2$ (we still require $m_{1} \geq 3$ ). Define the multi-graph $H^{*}$ by omitting all colors and arrows from 
$D_{p}(G)$ and shrinking every cycle representing the relation $g_{1}^{m_{1}}=e$ to a single vertex.

Theorem 14. If $H^{*}$ is bipartite, then

$$
\gamma(G) \leq 1+\frac{|G|}{4}\left(1-\frac{2}{m_{1}}-\frac{1}{k}\right) .
$$

Proof. Let $V_{1}\left(V_{2}\right)$ be the set of all elements $g$ of $G$ such that every word for $g$ involves $g_{2}$ an odd (even) number of times. Then $G=V_{1} \cup V_{2}$, since $H^{*}$ is bipartite. Select

$$
p_{g}= \begin{cases}\left(g g_{1}, g g_{2}, g g_{1}^{-1}\right) & \text { if } g \in V_{1}, \\ \left(g g_{1}^{-1}, g g_{2}, g g_{1}\right) & \text { if } g \in V_{2} .\end{cases}
$$

Computing orbits for this imbedding, we obtain

$$
r=r_{m_{1}}+r_{4 k}=\frac{|G|}{m_{1}}+\frac{|G|}{2 k} .
$$

We note that $p=|G|$ and $q=3|G| / 2$. Thus

$$
\gamma(G) \leq \gamma\left(D_{P}(G)\right) \leq 1+\frac{|G|}{4}\left(1-\frac{2}{m_{1}}-\frac{1}{k}\right) .
$$

Theorem 14 gives $\gamma(G)$ exactly, for $G=Z_{2} \times Z_{m}$ (m even), $S_{4}$, and $Z_{2} \times A_{4}$. The corollary below improves both bounds (given in Corollaries 11a and 12a) for $\gamma\left(S_{n}\right)$, for $n$ odd.

Corollary 14a. For $n$ odd,

$$
\gamma\left(S_{n}\right) \leq 1+\frac{(n-1) !}{6}(n-3), \quad n \geq 3 .
$$

Proof. Let $S_{n}=\langle r, s\rangle$, where $r=\left(\begin{array}{ll}1 & 2\end{array}\right)$ and $s=\left(\begin{array}{llll}1 & 2 & 3 & \cdots n\end{array}\right)$, so that $r^{2}=s^{n}=$ $\left(r^{-1} s^{-1} r s\right)^{3}=e$. We first claim that $H^{*}$ for $D_{P}\left(S_{n}\right)$ is bipartite. To see this, consider any cycle $C$ in $D_{P}\left(S_{n}\right)$; the cycle corresponds to $e$, which is in $A_{n}$. Since $n$ is odd, $s$ is also in $A_{n}$. Hence $r$ must appear an even number of times in $C$; thus all cycles in $H^{*}$ are even, and $H^{*}$ is bipartite. Thus Theorem 14 applies, with $m_{1}=n$ and $k=3$.

Asymptotic behavior. Let $\left\{G_{n}\right\}$ be a sequence of groups, with $G_{i}$ a proper subgroup of $G_{i+1}, i=1,2, \cdots$; let $j_{n}$ and $k_{n}$ be the degree and average number of sides per region for a genus presentation of $G_{n}$ respectively. Similarly, consider $\left\{G_{n}^{\prime}\right\}$ with parameters $j_{n}^{\prime}$ and $k_{n}^{\prime}$, where $G_{i}^{\prime}$ is a proper subgroup of $G_{i+1}^{\prime}$ and a subgroup of $G_{i}, i=1,2, \ldots$. Let $a(n)-b(n)$ indicate that $\lim _{n \rightarrow \infty}(a(n) / b(n))=1$; i.e. $a(n)$ is asymptotic to $b(n)$. Our final theorem relates the ratio $\gamma\left(G_{n}\right) / \gamma\left(G_{n}^{\prime}\right)$ to the index of $G_{n}^{\prime}$ in $G_{n}$, under certain conditions.

Theorem 15. If (i) $k_{n} \sim k_{n}^{\prime}$,

(ii) either $j_{n}-j_{n}^{\prime}$, or $j_{n} \rightarrow \infty$ and $j_{n}^{\prime} \rightarrow \infty$, and

(iii) $\gamma\left(G_{n}\right) \rightarrow \infty$ and $\gamma\left(G_{n}^{\prime}\right) \rightarrow \infty$, 
then

$$
\gamma\left(G_{n}\right) / \gamma\left(G_{n}^{\prime}\right)-\left(i_{n} / j_{n}^{\prime}\right)\left[G_{n}: G_{n}^{\prime}\right] .
$$

Proof. The euler formula $\gamma=1+1 / 2(q-p+r)$ applies for both $G_{n}$ and $G_{n}^{\prime}$, with $2 q=j p=k r$. Thus

$$
\begin{aligned}
\frac{\gamma\left(G_{n}\right)}{\gamma\left(G_{n}^{\prime}\right)} & =\frac{1+\frac{1}{2}\left(\frac{j_{n} p_{n}}{2}-p_{n}-\frac{j_{n} p_{n}}{k_{n}}\right)}{1+\frac{1}{2}\left(\frac{j_{n}^{\prime} p_{n}^{\prime}}{2}-p_{n}^{\prime}-\frac{j_{n}^{\prime} p_{n}^{\prime}}{k_{n}^{\prime}}\right)}=\frac{1+\frac{p_{n}}{2}\left(\frac{j_{n}}{2}-1-\frac{j_{n}}{k_{n}}\right)}{1+\frac{p_{n}^{\prime}}{2}\left(\frac{j_{n}^{\prime}}{2}-1-\frac{j_{n}^{\prime}}{k_{n}^{\prime}}\right)} \\
& \sim \frac{j_{n}}{j_{n}^{\prime}} \frac{p_{n}}{p_{n}^{\prime}}=\frac{j_{n}}{j_{n}^{\prime}}\left[G_{n}: G_{n}^{\prime}\right] .
\end{aligned}
$$

Corollary 15a. If, in fact, $j_{n}-j_{n}^{\prime}$, then

$$
\gamma\left(G_{n}\right) / \gamma\left(G_{n}^{\prime}\right) \sim\left[G_{n}: G_{n}^{\prime}\right]
$$

For example, let $G_{n}$ and $G_{n}^{\prime}$ be two elementary abelian 2-groups, of orders $2^{2 n}$ and $2^{n}$ respectively. By Theorem 6 ,

$$
\frac{\gamma\left(G_{n}\right)}{\gamma\left(G_{n}^{\prime}\right)}=\frac{1+2^{2 n-3}(2 n-4)}{1+2^{n-3}(n-4)} \sim 2^{n+1}=\frac{j}{j_{n}^{\prime}}\left[G_{n}: G_{n}^{\prime}\right] .
$$

Also, if equality holds in Corollaries $11 \mathrm{a}$ and $11 \mathrm{~b}$ (for $n$ even), we have an example of Corollary 15a, with $G_{n}=S_{2 n}, G_{n}^{\prime}=A_{2 n}$.

\section{REFERENCES}

1. L. W. Beineke and F. Harary, The genus of the n-cube, Canad. J. Math. 17 (1965), 494-496. MR 31 \#81.

2. G. Birkhoff and S. Mac Lane, Algebra, Macmillan, New York, 1967. MR 35 \#5266.

3. H. R. Brahana, Regular maps on an anchor ring, Amer. J. Math. 48 (1926), 225-240.

4. W. Burnside, Theory of groups of finite order, 2nd ed., Cambridge Univ. Press, Cambridge, 1911.

5. H. S. M. Coxeter and W. O. J. Moser, Generators and relations for discrete groups, 2nd ed., Ergebnisse der Mathematik und ihrer Grenzgebiete, N. F., Band 14, Springer-Verlag, Berlin and New York, 1965. MR 30 \#4818.

6. R. Dedekind, Über Gruppen, deren samtliche Teiler Normalteiler sind, Math. Ann. 48 (1897), 137-168.

7. W. Dyck, Gruppentheoretische Studien, Math. Ann. 20 (1882), 1-45.

8. J. Edmonds, A combinatorial representation for polyhedral surfaces, Notices Amer. Math. Soc. 7 (1960), 646. Abstract \#572-1.

9. F. Harary, Graph theory, Addison-Wesley, Reading, Mass., 1969. MR 41 \#1566.

10. H. Levinson, On the genera of graphs of group presentations, Intemat. Conf. on Combinatorial Math. (1970), Ann. New York Acad. Sci. 175 (1970), 277-284. MR 42 \#2968.

11. H. Maschke, The representation of finite groups, especially of the rotation groups of the regular todies in three-and four-dimensional space, by Cayley's color diagrams, Amer. J. Math. 18 (1896), 156-188. 
12. G. Ringel, Über drei kombinatorische Probleme am n-dimensionalen Würfel und Würfelgitter, Abh. Math. Sem. Univ. Hamburg 20 (1955), 10-19. MR 17, 772.

13. - Genus of graphs, Proc. Calgary Internat. Conf. Combinatorial Structures and Their Applications (Calgary, Alta., 1969), Gordon and Breach, New York, 1970, pp. 361-366. MR 42 \#7555.

14. A. T. White, The genus of repeated cartesian products of bipartite graphs, Trans. Amer. Math. Soc. 151 (1970), 393-404.

15. J. W. T. Youngs, Minimal imbeddings and the genus of a graph, J. Math. Mech. 12 (1963), 303-315. MR $26 \# 3043$.

DEPARTMENT OF MATHEMATICS, WESTERN MICHIGAN UNIVERSITY, KALAMAZOO, MICHIGAN 49001 